\title{
Quantum magnetism in the stripe phase: Bond versus site order
}

\author{
Jakub Tworzydło, ${ }^{*}$ Osman Y. Osman, Coen N. A. van Duin, and Jan Zaanen \\ Lorentz. Institute, Leiden University, P.O.B. 9506, 2300 RA Leiden, The Netherlands
}

(Received 17 August 1998)

\begin{abstract}
It is argued that the spin dynamics in the charge-ordered stripe phase might be revealing with regards to the nature of the anomalous spin dynamics in cuprate superconductors. Specifically, if the stripes are bond ordered much of the spin fluctuation will originate in the spin sector itself, while site-ordered stripes require the charge sector as the driving force for the strong quantum spin fluctuations. [S0163-1829(99)05301-1]
\end{abstract}

For quite some time it has been suspected ${ }^{1,2}$ that the anomalous spin dynamics of superconducting cuprates has to do with the $O(3)$ quantum nonlinear sigma model (QNLS), describing the collective dynamics of a quantum antiferromagnet. ${ }^{3}$ The discovery of the stripe phase ${ }^{4}$ opens a new perspective on these matters. ${ }^{5}$ Below the stripe-charge ordering temperature, charge fluctuations have to become inconsequential and the remaining spin dynamics should fall automatically in QNLS universality. As will be explained, the available data suggest that this spin dynamics is characterized by a close proximity to the QNLS zero-temperature transition. This enhancement of the quantum-spin fluctuations as compared to the half-filled antiferromagnet can have a variety of microscopic sources. Here we will focus on the possibility that these are due entirely to the charge-orderinginduced spatial anisotropy in the spin system. Although the influence of spatial anisotropy is well understood on the field-theoretic level, ${ }^{6,7}$ the charge can be bond ordered or site ordered $^{8}$ and this links the spin physics of the stripe phase to that of coupled spin ladders. ${ }^{9-11}$ At superconducting doping concentrations, bond and site order translate into coupled two-leg and three-leg spin ladders, respectively. We will present an in-depth quantitative analysis of both problems, showing that spatial spin anisotropy has to be largely irrelevant for site order, while it might well be the primary source of quantum spin fluctuations in the bond-ordered case. A strategy will be presented to disentangle these matters by experiment.

Let us first comment on the available information regarding the stripe-phase spin system. The spin-ordering temperature appears to be strongly surpressed as compared to half filling. ${ }^{4}$ A first cause can be a decrease of the microscopic exchange interactions. However, the more interesting possibility is that some microscopic disordering influence has moved the antiferromagnet closer to the zero-temperature order-disorder transition (quantum critical point). The few data available at present seem to favor the second possibility. We specifically refer to the ESR work by Kataev et al. ${ }^{12}$ on $\mathrm{La}_{1.99-x-y} \mathrm{Eu}_{y} \mathrm{Gd}_{0.01} \mathrm{Sr}_{x} \mathrm{CuO}_{4}$ exploiting the $\mathrm{Gd}$ local moments to probe the spin system in the $\mathrm{CuO}$ planes. Quite remarkably, little change is seen in the spin-lattice relaxation rate $\left(1 / T_{1}\right)$ at the charge-ordering temperature $T_{c o} \simeq 70 \mathrm{~K}$. Above $T_{c o}$ the $1 / T_{1}$ is quite similar to that in $\mathrm{La}_{2-x} \mathrm{Sr}_{x} \mathrm{CuO}_{4}$ where it is known from, e.g., neutron scattering that the mag- netic correlation length $\xi$ is already quite large at the temperatures of interest: since the width of the incommensurate peaks is smaller than their separation, the correlation length is larger than the stripe spacing. ${ }^{13}$ It follows that at $T \simeq T_{c o}$ a continuum description of the spin dynamics should be sensible. Below $T_{c o} 1 / T_{1}$ starts to increase exponentially upon lowering temperature, signaling the diverging correlation length associated with the renormalized classical regime. Taken together, this fits quite well the expectations for a quantum antiferromagnet that is rather close to its quantum critical point with a crossover temperature from the renormalized classical to the quantum critical regime $T^{*} \simeq T_{c o}$.

The increase of the coupling constant $g_{0}$, controlling the long wavelength fluctuations, originates in some microscopic phenomenon. A limiting case is that charge can be regarded as completely static even on the scale of the lattice constant, such that its effect is to cause a spatially anisotropic distribution of exchange interactions. ${ }^{6,7}$ As indicated in Fig. 1, there are two options: ${ }^{8}$ the stripes can be bond or site ordered. It is expected that the spin dynamics associated with the hole-rich regions is characterized by a short time scale and the magnetic ordering phenomena are therefore associated with the magnetic domains. The spin-only model of relevance becomes either a spin $S=1 / 2$ Heisenberg model describing three-leg ladders (site ordered) or two-leg ladders (bond ordered) with uniform exchange interactions $(J)$, mutually coupled by a weaker exchange-interaction coupling $(\alpha J, \alpha<1)$. This model is explicitly,

$$
\mathcal{H}=J \sum_{\overrightarrow{\mathbf{i}}} \mathbf{S}_{\mathbf{i}} \mathbf{S}_{\mathbf{i}+\delta_{y}}+J \sum_{i_{x} \neq p n_{l}, i_{y}} \mathbf{S}_{\mathbf{i}} \mathbf{S}_{\mathbf{i}+\delta_{x}}+\alpha J \sum_{i_{x}=p n_{l}, i_{y}} \mathbf{S}_{\mathbf{i}} \mathbf{S}_{\mathbf{i}+\delta_{x}},
$$
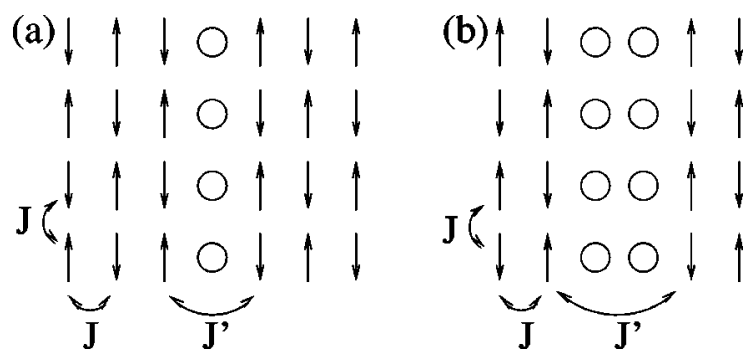

FIG. 1. Schematic distinction between site-ordered (a) and bond-ordered (b) stripes. 
where $\mathbf{i}=\left(i_{x}, i_{y}\right)$ runs over a square lattice, $\boldsymbol{\delta}_{x}=(1,0), \quad \boldsymbol{\delta}_{y}$ $=(0,1) . \quad n_{l}$ measures the width of the ladder and $p$ counts the ladders.

Since the interest is in nonuniversal quantities as related to the nontrivial lattice cutoff, we studied the model equation (1) numerically using a highly efficient loop algorithm quantum Monte Carlo method ${ }^{14}$ supported with a technique of improved estimators. ${ }^{15}$ To keep track of the various finitetemperature crossovers we focused on the temperature dependence of the staggered correlation length in both directions, parallel $\left(\xi_{y}\right)$ and perpendicular $\left(\xi_{x}\right)$, to the stripes. We typically insisted on $3 \times 10^{4}$ loop updates for equilibration and $(2-3) \times 10^{5}$ updates for a measurement, keeping the dimensions of the system in the $x$ and $y$ directions $L_{x, y}$ $\geqslant 6 \xi_{x, y}$, to avoid finite-size effects. ${ }^{16}$ The correlation length was determined by fitting the staggered spin-spin correlation function $C(\mathbf{r})=(-1)^{r_{x}+r_{y}}\left\langle\mathbf{S}_{i+r} \cdot \mathbf{S}_{i}\right\rangle$, using a symmetrized two-dimensional Ornstein-Zernike form $C(r)$ $=A\left(r^{-1 / 2} e^{-r / \xi}+(L-r)^{-1 / 2} e^{-(L-r) / \xi}\right)$ separately for the $x\left[\mathbf{r}=(r, 0), L=L_{x}\right]$ and $y\left[\mathbf{r}=(0, r), L=L_{y}\right]$ directions, omitting the first few points to ensure asymptoticity. We checked our results against the known results for both isolated ladders by Greven et al. ${ }^{17}\left(\alpha=0, \quad n_{l}=1,2,3\right)$ and the lowtemperature results for the isotropic $(\alpha=1)$ limit. ${ }^{15,16,18}$

Since $O(3)$ universality is bound to apply at scales much larger than any lattice-related crossover scale, universal forms for the temperature dependence of the correlation length can be used to further characterize the longwavelength dynamics. The absolute lattice cutoff is reached at a temperature $\left(T_{\max }\right)$ where the correlation length parallel to the stripes $\left(\xi_{y}\right)$ becomes of order of the lattice constant. However, the problem is characterized by a second cutoff: when the correlation length is less than the lattice constant in the direction perpendicular to the stripes $\left(a_{x}\right)$, the dynamics is that of $N_{x}$ independently fluctuating spin ladders. We define $T_{0}$ as the temperature where $\xi_{x} \simeq a_{x}$ is the crossover temperature below which the system approaches $(2+1)$ dimensional $O(3)$ universality. In this latter regime, further crossovers are present. When the effective coupling constant $\left(g_{0}\right)$ is less than the critical coupling constant $\left(g_{c}\right)$ a crossover occurs from a "high"-temperature quantum critical (QC) to a low-temperature renormalized classical (RC) regime. In the QC regime $\xi \sim 1 / T$ while the crossover temperature $T^{*}$ to the RC regime can be deduced from the exponential increase of the correlation length at low $T$, using ${ }^{3,16,19}$

$$
\xi(T) \propto \frac{e^{T^{*} / T}}{2 T^{*}+T},
$$

where $T^{*}=2 \pi \rho_{s}$ in terms of the spin stiffness $\rho_{s}(\alpha)$. When $g_{0}>g_{c}$, the ground state is quantum disordered (QD) as signaled by $\xi$ becoming temperature independent, and the crossover temperature $T^{\prime}$ between the QC and QD regimes is estimated from the approximate relation ${ }^{17}$

$$
T^{\prime}=\frac{c_{y}}{\xi_{y}(T \rightarrow 0)},
$$

where $c_{y}$ is spin-wave velocity in the strong direction.

We determined the various crossover lines as function of $\alpha$ for the cases $n_{l}=1,2$, and 3 (anisotropic Heisenberg,

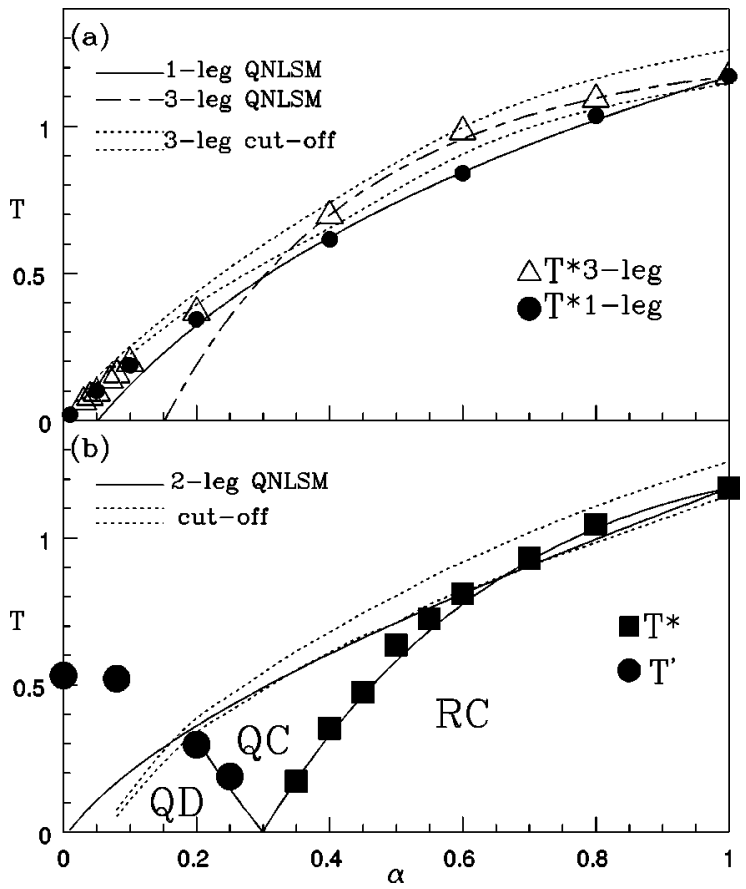

FIG. 2. Crossover temperatures as a function of anisotropy $\alpha$ for the coupled three-leg (a) and two-leg (b) spin-ladder models. The lines and points refer to the analytical and numerical results, respectively, for the various scales. Notice that the one-leg "cutoff', (onedimensional to two-dimensional crossover) follows closely the results for $T^{*}$.

coupled two- and three-leg ladders, respectively). To determine $T_{0}$, we used for $\alpha$ close to 1 the same criterion as for the $T_{\max }$ determination in the isotropic problem $\left[\xi_{x}\left(T^{0}\right)\right.$ $=0.7-0.8]$. This becomes inconsistent for small $\alpha$ where one better incorporates the width of the ladder $\left[\xi_{x}\left(T_{0}\right)=n_{l}\right.$ $\times(0.7-0.8)]$ and we used a linear interpolation to connect smoothly both limits. We checked that below the $T_{0}$, determined in this way, both $\xi_{x}$ and $\xi_{y}$ exhibited the same dependence on temperature after an overall change of scale, demonstrating that the collective dynamics is indeed in a $(2+1)$ dimensional regime.

In Fig. 2 we summarize our results in the form of a crossover diagram as function of $\alpha$ and temperature, both for the one- and three-leg [Fig. 2(a)] and the two-leg [Fig. 2(b)] cases. Consistent with analytic predictions, ${ }^{10}$ the behavior is radically different for the half-integer spin one- and three-leg cases on the one hand, and the "integer spin" two-leg case on the other hand. Let us first discuss the former. Here the ground state remains in the renormalized classical regime for any finite $\alpha$. The reason is obvious. In isolated ladders ( $\alpha$ $=0$ ) with an uneven number of legs the ground state is a Luttinger liquid exhibiting algebraic long-range order and any finite ladder-to-ladder interaction will suffice to stabilize true long-range order at $T=0 .{ }^{10,11}$ This in turn implies a finite $T^{*}$ where the classical nature of the ground state becomes visible. Interestingly, our calculations indicate that $T^{*}$ and $T^{0}$ basically coincide for any $\alpha$ : at the moment the system discovers that it is $2+1$ dimensional, the classical behavior sets in. Our finding that $T^{0}$ increases linearly with $\alpha$ for small $\alpha$ [Fig. 2(a)] confirms the scaling theory by Affleck and Halperin for this problem. ${ }^{11}$ The behavior of the spin- 
spin correlator for an isolated chain $\langle S(x) S(0)\rangle$ $\sim(1 / x) \exp \left(-x / \xi_{1}\right), \quad \xi_{1} \sim 1 / T$ signals the approach to the Gaussian fixed point: within the thermal length $\xi_{1}$ the system exhibits algebraic long-range order. For finite $\alpha$ the crossover temperature $T^{0}$ can be found using the standard meanfield consideration: at $T^{0}$ temperature becomes of order of the exchange interaction between two patches of correlated spin on neighboring chains of size $\xi_{1}: k_{B} T^{0}$ $\simeq \alpha \Phi_{1}^{2} / \xi_{1}\left(T^{0}\right)$, where $\Phi_{1}=\xi_{1}\left(T^{0}\right) \phi, \quad \phi$ being the microscopic staggered magnetization. Taking $\phi$ independent of $\alpha$ would yield the erroneous result that $T^{0} \sim \sqrt{\alpha}$. The subtlety is that when $\alpha$ is sufficiently small, the quantum dynamics within the correlation volume $\xi_{1}$ is already in the $(2+1)$ dimensional regime. ${ }^{7}$ Using the $T=0$ result by Affleck and Halperin that $\phi \sim \sqrt{\alpha},{ }^{11}$ we recover $T^{0} \sim \alpha, \quad \alpha \ll 1$. The other feature worthwhile mentioning is that $T^{0}$ and $T^{*}$ are identical for the one- and three-leg cases for small $\alpha$ 's. This is in line with the observations by Frishmuth et al. ${ }^{20}$ that these spin ladders renormalize in identical Luttinger liquids when the ladder exchange interactions are isotropic.

In the two-leg ladders case [Fig. 2(b)] the quantum orderdisorder transition occurs at a finite value of $\alpha, \alpha_{c}$ $=0.30(2)$. This is in line with the qualitative expectations (see also Ref. 21) and agrees with the quantitative value obtained in a different context. ${ }^{22}$ Since the isolated two-leg ladders are incompressible spin systems, the ladder-to-ladder interaction has to overcome the single-ladder energy gap before the two-dimensional lock-in can occur. This critical $\alpha$ is rather large, and in addition, the $(1+1)$-dimensional $\rightarrow(2$ +1 )-dimensional crossover temperature $T^{0}$ shows the upward curvature $\left(T^{0} \sim \sqrt{\alpha}\right)$ previously predicted from a scaling analysis of the anisotropic QNLS model (AQNLS). ${ }^{7}$ As a ramification, $T^{0}$ and $T^{*}$ (as well as $T^{\prime}$ ) separate and a large, genuinely $(2+1)$-dimensional quantum critical regime opens up around $\alpha_{c}$. This is in marked contrast with the isotropic Heisenberg model where the renormalized classical regime sets in essentially at the lattice cutoff. ${ }^{23,24}$

The gross $\alpha$ dependences of the various crossover temperatures can be understood by considering the AQNLS model obtained by taking the naive continuum limit for the ladder problem. An average staggered field $\boldsymbol{\phi}$ is introduced for a block of $2 \times n_{l}$ sites. Integrating out the quadratic fluctuations, ${ }^{25}$ the effective action for $\boldsymbol{\phi}$ becomes the AQNLS model with anisotropic spin-wave velocities,

$$
\begin{gathered}
c_{x}^{2}=\alpha c_{0}^{2}\left\{\begin{array}{l}
\frac{(3+\alpha)}{2(1+\alpha)} \quad \text { for } n_{l}=2, \\
\frac{9(7+3 \alpha)}{2(1+2 \alpha)(13+2 \alpha)} \quad \text { for } n_{l}=3,
\end{array}\right. \\
c_{y}^{2}=c_{0}^{2} \begin{cases}\frac{(3+\alpha)}{4} & \text { for } n_{l}=2, \\
\frac{3(7+3 \alpha)}{2(13+2 \alpha)} & \text { for } n_{l}=3,\end{cases}
\end{gathered}
$$

where $c_{0}$ is the spin-wave velocity in the isotropic limit. The coupling constant $g_{0}$ is $\alpha$ independent and the same as for

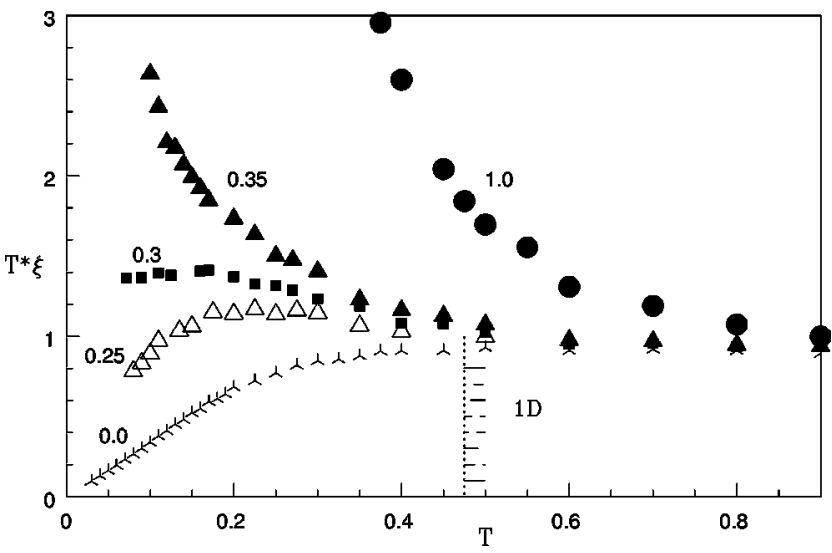

FIG. 3. $\xi_{y} T$ vs temperature for the two-leg system, when the $\alpha$ 's are close to critical point. Results for $\alpha=0.0$ (isolated ladders) and 1.0 (isotropic limit) are added for comparison. The vertical bar indicates the one-dimensional to two-dimensional crossover temperature.

the isotropic model. According to the scaling analysis of Ref. 7 , the renormalized spin stiffness becomes in terms of the velocities $c_{x, y}$,

$$
\rho_{s}(\alpha)=\rho_{s} \frac{c_{x}\left(1-\frac{g_{0}}{g_{c}(\alpha)}\right)}{c_{y}\left(1-\frac{g_{0}}{g_{c}(1)}\right)},
$$

where

$$
\begin{aligned}
g_{c}(\alpha)= & 4 \pi \sqrt{c_{0} / c_{y}}\left(1+\frac{2}{\pi}\left\{c_{y} \operatorname{arcsinh}\left[c_{x} / c_{y}\right] / c_{x}\right.\right. \\
& \left.\left.+\ln \left[c_{y}\left(1+\sqrt{1+c_{x}^{2} / c_{y}^{2}}\right) / c_{x} /(1+\sqrt{2})^{2}\right]\right\}\right)
\end{aligned}
$$

and $\rho_{s}$ is the spin stiffness for $\alpha=1$. According to Ref. 7, the crossover scales are $T^{*}=2 \pi \rho_{s}(\alpha)$, $T^{0}=2 \pi \rho_{s} c_{x}\left[g_{0} /\left(4 \pi c_{0}\right)+\left(1-g_{0} / g_{c}\right) / c_{y}\right] \quad$ and $T^{\prime}$ $=$ const $\left|\rho_{s}(\alpha)\right|$. It turns out that for the bare coupling constant $g^{0}$ as determined for the isotropic case $\left(g^{0}=9.1\right)$, the order-disorder transition occurs at a somewhat small value of $\alpha=0.08$, which is not surprising given the approximations involved (one-loop level). However, by adjusting $g_{0}$ to shift $\alpha_{c}$ to its numerical value $\left(g_{0}=11.0\right)$, we find a very close agreement between the numerical and analytical results for the various crossover temperatures [Fig. 2(b)]. As can be seen from Fig. 2(a), the above analysis also works quite well for the three-leg ladders for $\alpha \geqslant 0.4$. Remarkably, it seems that $T^{*}$ switches rather suddenly from the AQNLS behavior at large $\alpha$ to the linear behavior expected for the Luttinger liquid regime, as if the topological terms start to dominate rather suddenly.

Besides its intrinsic interest, the above does have potentially important ramifications for the understanding of the quantum magnetism in cuprates: bond ordering of stripes would imply that already at rather moderate values of the anisotropy $\alpha$, spin-ladder physics alone would enhance the quantum spin fluctuations substantially. This can be further illustrated by comparing the temperature dependence of 
$T \xi_{y}(T)$ for the isotropic spin system $\alpha=1$ with that of the coupled two-leg ladders in the vicinity of the critical $\alpha$ (Fig. 3 ). This quantity can be directly compared with the spin-spin relaxation rate $1 / T_{2 G}$ and, with some caution, also to $1 / T_{1}$ (Refs. 23 and 26) (a dynamical critical exponent $z=1$ is only strictly obeyed in the QC regime). As compared to the isotropic case, the exponential increase of $T \xi$ (signaling the renormalized classical regime) is shifted to a low temperature, while over most of the temperature range $T \xi(T)$ is constant, as is found in cuprates. It is noted that the "quantumcritical signature" $\xi \sim 1 / T$ extends in the temperature range above the dimensional crossover temperature $T^{0}$. Since this regime is nonuniversal this should be regarded as a quasicriticality.

This is no more than suggestive. However, it points at a simple strategy to clear up these matters by experiments in- volving the static stripe phase. It should be established if the stripe phase is site or bond ordered, which can be done by NMR. Next, the $\alpha$ should be determined from neutron measurements of the spin-wave velocities, Eq. (5). Using these as an input, the temperature dependence of the correlation length, as well as the NMR relaxation rates, can be calculated to a high precision starting from a microscopic spinonly dynamics. Comparison of these quantities to experiment should yield insights into the microscopic origin of the peculiar spin dynamics in doped cuprates.

We thank B. I. Halperin for helpful discussions. J.T. acknowledges support from the Foundation for Polish Science (FNP), and J.Z. financial support by the Dutch Academy of Sciences (KNAW).
*On leave from Institut of Theoretical Physics, Warsaw University.

${ }^{1}$ A. V. Chubukov, S. Sachdev, and J. Ye, Phys. Rev. B 49, 11919 (1994); A. V. Chubukov, S. Sachdev, and A. Sokol, ibid. 49, 9052 (1994).

${ }^{2}$ A. Sokol and D. Pines, Phys. Rev. Lett. 71, 2813 (1993).

${ }^{3}$ S. Chakravarty, B. I. Halperin, and D. R. Nelson, Phys. Rev. Lett. 60, 1057 (1988); Phys. Rev. B 39, 2344 (1989).

${ }^{4}$ J. M. Tranquada et al., Nature (London) 375, 561 (1995); J. M. Tranquada, Physica B 241-243, 745 (1998).

${ }^{5}$ G. Aeppli, T. E. Mason, S. M. Hayden, H. A. Mook, and J. Kulda, Science 278, 1432 (1998).

${ }^{6}$ A. H. Castro Neto and D. Hone, Phys. Rev. Lett. 76, 2165 (1996).

${ }^{7}$ C. N. A. van Duin and J. Zaanen, Phys. Rev. Lett. 80, 1513 (1998).

${ }^{8}$ J. Zaanen and O. Gunnarsson, Phys. Rev. B 40, 7391 (1989); S. R. White and D. J. Scalapino, ibid. 55, R14 701 (1997); J. Zaanen, cond-mat/9711009, J. Phys. Chem. Solids (to be published).

${ }^{9}$ E. Dagotto and T. M. Rice, Science 271, 618 (1996).

${ }^{10}$ Z. Wang, Phys. Rev. Lett. 78, 126 (1997).

${ }^{11}$ I. Affleck and B. I. Halperin, J. Phys. A 29, 2627 (1996).

${ }^{12}$ V. Kataev et al., Phys. Rev. B 55, R3394 (1997).

${ }^{13}$ G. Aeppli et al., Science 278, 1432 (1997).

${ }^{14}$ H. G. Evertz, G. Lana, and M. Marcu, Phys. Rev. Lett. 70, 875 (1993); H. G. Evertz and M. Marcu, in Quantum Monte Carlo
Methods in Condensed Matter Physics, edited by M. Suzuki (World Scientific, Singapore, 1994).

${ }^{15}$ U. J. Wiese and H.-P. Ying, Z. Phys. B 93, 147 (1994); Phys. Lett. A 168, 143 (1992).

${ }^{16}$ B. B. Beard, R. J. Birgeneau, M. Greven, and U. J. Wiese, Phys. Rev. Lett. 80, 1742 (1998); J. K. Kim and M. Troyer, ibid. 80, 2705 (1998).

${ }^{17}$ M. Greven, R. J. Birgeneau, and U. J. Wiese, Phys. Rev. Lett. 77, 1865 (1996).

${ }^{18}$ B. B. Beard and U. J. Wiese, Phys. Rev. Lett. 77, 5130 (1996).

${ }^{19}$ P. Hasenfratz and F. Niedermayer, Phys. Lett. B 268, 231 (1991); Z. Phys. B 92, 91 (1993).

${ }^{20}$ B. Frischmuth, B. Ammon, and M. Troyer, Phys. Rev. B 54, R3714 (1996); B. Frischmuth, S. Haas, G. Sierra, and T. M. Rice, ibid. 55, R3340 (1997).

${ }^{21}$ S. Gopalan, T. M. Rice, and M. Sigrist, Phys. Rev. B 49, 8901 (1994); M. Azzouz, B. Dumoulin, and A. Benyoussef, ibid. 55, R11 957 (1997).

${ }^{22}$ M. Imada and Y. Iino, J. Phys. Soc. Jpn. 66, 568 (1997).

${ }^{23}$ A. W. Sandvik and D. J. Scalapino, Phys. Rev. B 51, R9403 (1995).

${ }^{24}$ M. S. Makivić and H.-Q. Ding, Phys. Rev. B 43, 3562 (1991).

${ }^{25}$ D. Senechal, Phys. Rev. B 52, 15319 (1995).

${ }^{26}$ A. W. Sandvik, E. Dagotto, and D. J. Scalapino, Phys. Rev. B 53, R2934 (1996). 